\title{
New Approach on Analysis of Pathologic Cardiac Murmurs Based on WPD Energy Distribution
}

\author{
Zhongwei Jiang $^{1 *}$, Ting Tao ${ }^{1}$ and Haibin Wang ${ }^{2}$ \\ ${ }^{1}$ Graduate School of Science and Engineering, Yamaguchi University, Yamaguchi, \\ Japan, \\ ${ }^{2}$ School of Electrical and Information Engineering, Xihua University, Chengdu, China
}

Submitted March 2014. Accepted for publication September 2014.

\begin{abstract}
In this paper, an approach on analysis of the pathologic cardiac murmurs for congenital heart defects was proposed based on the wavelet packet (WP) technique. Considering the difference of the energy intensity distributions for the innocent and pathologic murmurs in frequency domain, the WP decomposition was introduced and the WP energies at each frequency band were calculated and compared. Based on the analysis of a large amount of clinic heart sound data, the murmurs energy distributions were divided into five frequency bands, and the relative evaluation indexes for cardiac murmurs (ICM) were proposed for analysis of the pathologic murmurs. Finally, the threshold values between the innocent and pathologic cardiac murmurs were determined based on the statistical results of the normal heart sounds. The analysis results validate the proposed evaluation indexes and the corresponding thresholds.
\end{abstract}

Keywords: cardiac murmurs analysis, energy distribution, wavelet decomposition, evaluation index, congenital heart defects

\section{INTRODUCTION}

Congenital heart defects (CHDs) are problems with the heart's structure or function that is formed before birth [1], with a rate of eight out of every 1,000 newborns. It is known that this kind of defects can be cured with a high probability if the diseases could be detected in an early stage. Therefore, the research on the early detection of CHDs is one of the most important medical research areas [2]. There are many types of congenital heart defects. Some are simple and some are more complex. Atrial septal defect (ASD) is one of the CHDs, a hole in the part of the septum that separates the atria. The hole makes oxygen-rich blood from the left atrium to flow into the right atrium, instead of flowing into the left ventricle as it should. ASDs can be small, medium, or large. Medium and large ASDs allow more blood to leak from

*Corresponding author: Zhongwei Jiang, Tokiwadai 2-16, Ube, Yamaguchi, 755-8611, Japan. Phone: (81)836 85 9137. Fax: (81)836 85 9101. E-mail: jiang@yamaguchi-u.ac.jp. Other authors: s503wc@yamaguchi-u.ac.jp; 657189096@qq.com. 
one atrium to the other. Ventricular septal defect (VSD) is another type of CHD, with a hole in the part of the septum that separates the ventricles. Small VSDs do not cause problems and may close on their own. However, large VSDs allow much blood to flow from the left ventricle to the right ventricle. As a result, the left side of the heart must work harder than normal and extra blood flow increases blood pressure in the right side of the heart and the lungs. Furthermore, the most common complex heart defect is tetralogy of Fallot (TOF), which is a combination of four defects: Pulmonary valve stenosis, a large VSD, an overriding aorta and right ventricular hypertrophy. ASD, VSD and TOF account for the majority of the CHD [3].

Heart murmurs are pathological sounds produced by turbulent blood flow due to certain cardiac defects, and they are the most common reasons for referral to the pediatric cardiologist. In children, about $50-70 \%$ of these murmurs are clinically insignificant [4], but if the child is crying, uncooperative to the examiner or breathing loudly, some other murmurs may occur. Because of the difficulty of mastering auscultation skills, innocent and organic heart murmurs cannot be readily distinguished. Therefore, heart murmur quantitative analysis is necessary. Recently, the demand for evaluation of the murmurs from auscultation of cardiac sounds has been addressed by researchers and clinicians [5-8]. To detect and analyze the heart sound (HS) murmurs, many approaches have been carried out, including a multivariate matching pursuit method [7] used to model the murmurs by decomposing them into a series of parametric time-frequency atoms, and then the model parameters used to identify the cardiac sound signals. An adaptive singular spectrum analysis approach [8] was applied to the changes in the statistical properties of the sound data for detection of murmurs.

Since the heart murmurs show clearly different characteristics in the frequency domain compared with the time domain, many researchers have focused on the characteristic extraction by local frequency analysis method, such as the wavelet decomposition (WD) or WPD [9-14, 23], the neural network [6, 15-18], support vector machine(SVM) [19-21]. Sepehri, et al. [18], studied a method for automatic screening of CHD in children with neural network classifier. The pathological murmurs of CHD were identified by examining the HS energy over special frequency bands called ArashBands. However, the Arash-Bands determined for each CHD in the study were overlapped with the frequencies 16-121 Hz. Based on our study, the main energy distributions either for normal or abnormal HSs are concentrated in the frequency range of $10-100 \mathrm{~Hz}$. We also found that frequency band is much influenced by measurement situations, ages, body types, mixed heart defects, etc. We have tried many methods but it seems difficult to identify some murmurs by the Arash-Bands. Furthermore, Samjin Choi, et al. [9, 23] proposed insufficiency murmur identification and valvular disorders detection using wavelet packet decomposition, and HS signals with frequency range 20$700 \mathrm{~Hz}$ were preferred; furthermore, the features were extracted from WP coefficient calculated for each subdivision. However, the subjects in these two studies were from the medical text book [26] or internet web [27]. It is not clear whether WP coefficient 
calculated for each subdivision with the frequency range $20-700 \mathrm{~Hz}$ can identify the CHD signals in general auscultation environment in hospital.

In our study, a novel approach is investigated for analysis of the pathologic cardiac murmurs of CHD with the energy distributions over special frequency bands. The clinical CHD including the most common CHD of children (3 month to 10 years old, several are 24 27 years old) is investigated. In collecting the clinical signals from children in hospital, we found that children's thoracic cavities are very thin, and during clinical auscultation, high frequency $(344-1378 \mathrm{~Hz}) \mathrm{HS}$ components produced by blood flow through the valves are easy to collect through the thin chest, and the heart beat activity always cause chest vibration through the thin chest. Hence, the very low frequency $(5-21 \mathrm{~Hz})$ component from chest vibration or body movement can be collected. Therefore, in our study, we preferred the heart signals with the frequency range with $5-1378 \mathrm{~Hz}$. According to the analysis results, we also found that frequency division too small would highlight breathe sound component and other noises from body moving or environment, which would influence the identification result. Therefore, in our study five frequency band divisions were employed; the wavelet packet decomposition (WPD) is introduced and the WP energy distributions at these five frequency bands are calculated and compared. The evaluation indexes for cardiac murmurs are proposed for the analysis of the pathologic murmurs. Finally, the threshold values between the innocent and pathologic murmurs are determined based on the statistical results of the HSs. The analysis results demonstrate the validation and efficiency by using the proposed evaluation indexes and the corresponding thresholds.

\section{METHOD}

Auscultation denotes the act of analyzing sounds from the body, which is produced by the mechanical vibrations generated in the organs. The HSs are primarily generated from blood turbulence. Heart defects can cause heart murmurs (extra or unusual sounds heard during a heartbeat). Doctors can hear heart murmurs using a stethoscope. In CHD patients, a pulmonary ejection click or/and soft and scratchy systolic ejection murmurs are audible [22]. HS auscultation is usually performed at four locations (aortic, pulmonic, tricuspid and mitral areas) in front of the chest. In this paper, the cardiac sound signals auscultated from the pulmonic area were selected as the analysis signals because the HS is most audible in this area. The use of the data in this study has been approved by local data collection ethics committee.

The cardiac murmurs for different heart defects usually contain different frequency components. If the sound signal can be decomposed in different requested frequency ranges in a suitable way, the corresponding energy intensities can be evaluated quantitatively. In this study, the cardiac signal is decomposed and reconstructed at each requested frequency band by the WPD technique and their murmurs energy intensities are calculated and used for the cardiac murmurs evaluation. The block diagram for murmurs analysis is depicted in Figure 1 and the detail is described below. 


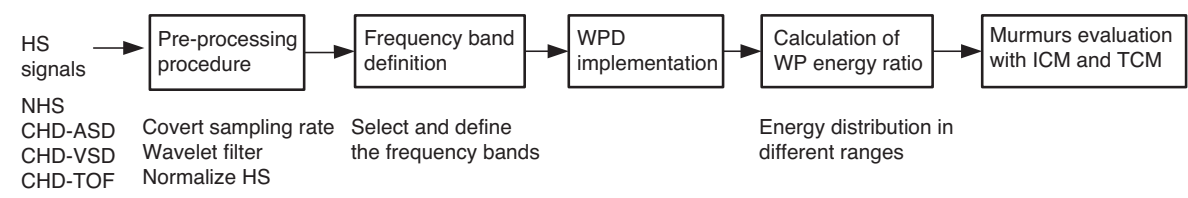

Figure 1. The block diagram for murmurs analysis.

\subsection{Pre-processing}

The original HS signals were recorded by an electric stethoscope with 16-bit accuracy and $44.1 \mathrm{kHz}$ sampling frequency. The recorded signal $x(i)$ is first down-sampled to $11025 \mathrm{~Hz}$ to reduce the calculation time. Next, WPD implementation is applied to the signal with MATLAB software. Daubechies 10 (DB10) wavelet was used as the mother wavelet because it very well addresses biomedical signals [9, 20, 24]. The original signal $x(i)$ is first preprocessed by the WPD-based filter with frequency band of 5.381378.1 Hz. The obtained signal $x_{m}(i)$ is then normalized by Eqn. (1) and used in the following analysis:

$$
\hat{x}(i)=\frac{x_{m}(i)}{\max \left(x_{m}(i)\right)}
$$

\subsection{Frequency Band Definition}

By considering the frequency ranges of the innocent and pathologic murmurs in the frequency domain, in this study, the wavelet packet decomposition at level 10 is employed to split frequency bandwidths of HS signals. Through clinical auscultation observation and energy intensities analysis at each level (d10 d3), the interested parts and useful combinations are implemented, and five frequency bands are defined and shown in Table 1; they are named as very low frequency (VLF), standard frequency (SF), low frequency (LF), middle frequency (MF) and high frequency (HF). Their corresponding wavelet detail coefficient levels and center frequencies are also presented in Table 1.

Table 1. Murmurs frequency bands definition.

\begin{tabular}{lccccc}
\hline Frequency band number $(j)$ & $\mathbf{1}$ & $\mathbf{2}$ & $\mathbf{3}$ & $\mathbf{4}$ & $\mathbf{5}$ \\
\hline Frequency band & $\mathrm{VLF}$ & $\mathrm{SF}$ & $\mathrm{LF}$ & $\mathrm{MF}$ & $\mathrm{HF}$ \\
Wavelet detail coefficients & $\mathrm{d} 10+\mathrm{d} 9$ & $\mathrm{~d} 8+\mathrm{d} 7$ & $\mathrm{~d} 6$ & $\mathrm{~d} 5$ & $\mathrm{~d} 4+\mathrm{d} 3$ \\
Frequency band & & & & & \\
corresponded & & & & & \\
to sampling & & & & & \\
frequency (Hz) & $5.38-21.53$ & $21.53-86.1$ & $86.1-172.2$ & $172.2-344.4$ & $344.4-1378$ \\
Center frequency (Hz) & $7-14$ & $29-59$ & 117 & 235 & $471-942$ \\
(Measured center frequency) & $(6-12.5)$ & $(25-50)$ & $(100)$ & $(200)$ & $(400-800)$ \\
\hline
\end{tabular}




\subsection{WPD Implementation}

The wavelet packet analysis is an extension of the discrete wavelet transform (DWT) and is one of the most efficient decomposition that could be performed on the signal. Daubechies type wavelet (DB10) is used to build a wavelet filter. The center frequencies are calculated from the mother wavelet (DB10) at the corresponding detail levels (Table 1). Further the reconstructed signals at each decomposition level are applied by FFT and their peak frequencies are measured and named as measured center frequency (Table 1) just for reference. Figure 2 shows the plots of some typical HSs treated by WP analysis method. Figure 2(a)-(d) are the examples of NHS, ASD, VSD and TOF signals obtained by reconstruction of the components at VLF, SF, LF, MF and HF band as defined in Table 1. A simple sum of all the VLF, SF, LF, MF and HF components represent the original HS signal.

The NHS signal plots at each frequency band show the $1^{\text {st }}$ and $2^{\text {nd }}$ sounds clearly and the energy is mainly distributed at the SF band. As for ASD, the signal plots show that the murmurs appeared mainly at LF and MF bands. As for VSD and TOF, the murmurs have stronger energy distributions at MF and HF frequency bands.

\subsection{WP Energy (WPE) Ratios}

Figure 2(a)-(d) show that the energy of all types of HS signals is mainly concentrated at SF band. Further, compared to NHS, CHD signals have higher energy intensities at the higher frequency bands. To quantify the cardiac murmurs, WP energy ratio $\hat{E}_{j}$ is proposed and defined as the following:

$$
\hat{E}_{j}=\frac{E_{j}}{E_{\text {total }}} \times 100 \%, \text { where } E_{j}=\sum_{i=1}^{N}\left|\hat{x}_{j}(i)\right|^{2} \text { and } E_{\text {total }}=\sum_{j=1}^{n} E_{j}
$$

$\hat{x}_{j}(i)$ is the reconstructed signal at the corresponding frequency band $j, N$ is the signal length and $j=1,2,3,4,5$ are corresponding to frequency bands VLF, SF, LF, MF and $\mathrm{HF}$ respectively.

\section{DATA ANALYSIS}

The original HS signals were recorded by an electric stethoscope with 16-bit accuracy and $44.1 \mathrm{kHz}$ sampling frequency. HS data were collected from 85 test subjects who have signed an informed consent. The subjects included 21 healthy young college students and 64 CHD patients. CHDs patients included 17 ASD, 29 VSD and 18 TOF.

The energy distributions for these four conditions (NHS, ASD, VSD and TOF) are calculated at each frequency band defined in Table 1. The energy distribution histograms at SF, LF, MF and HF frequency bands are plotted in Figure 3 to Figure 6. The $y$-axis shows the number of samples or frequency of the observations in the interval and the x-axis is the energy ratio $\hat{E}_{j}$ in percentage. There are about $40 \%$ to $90 \%$ energy distributed at SF frequency band, $5 \%$ to $30 \%$ at $\mathrm{LF}$ band, $1 \%$ to $10 \%$ at $\mathrm{MF}$ and $0.1 \%$ to $4 \%$ at $\mathrm{HF}$ band. 

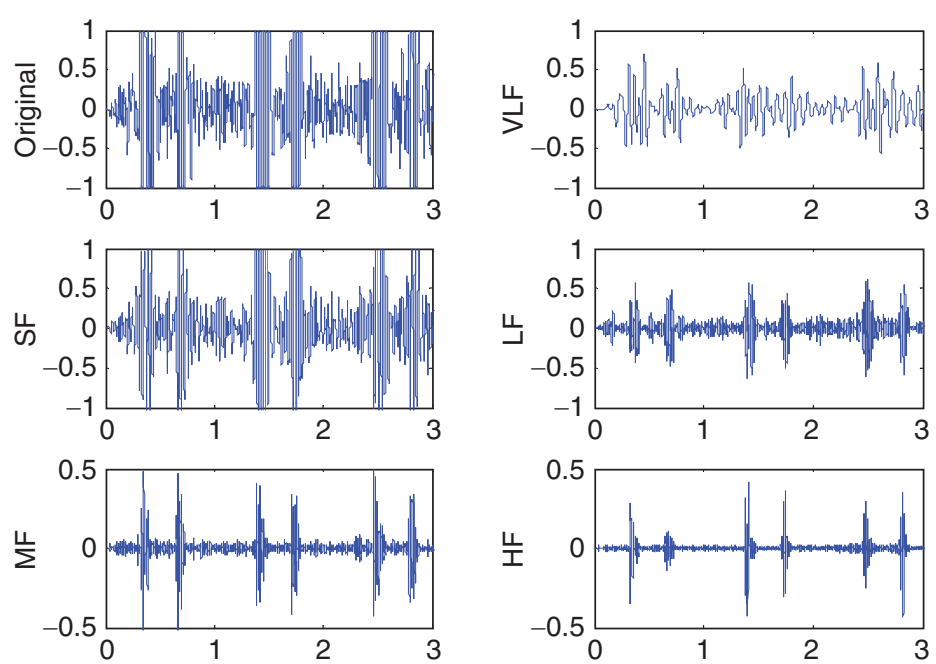

(a) NHS
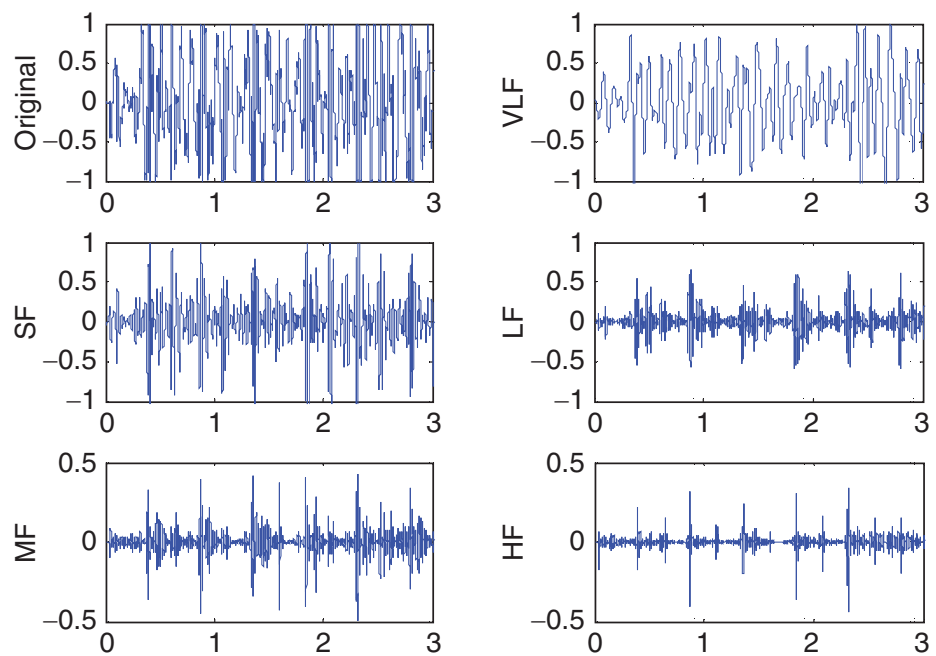

(b) ASD

Figure 2. (Continued) 

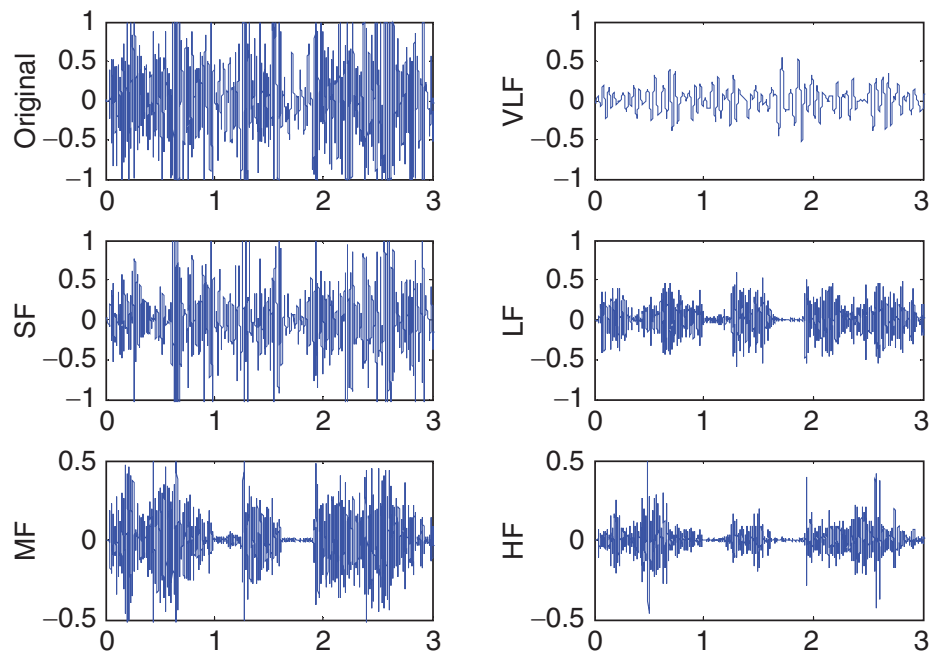

(c) VSD
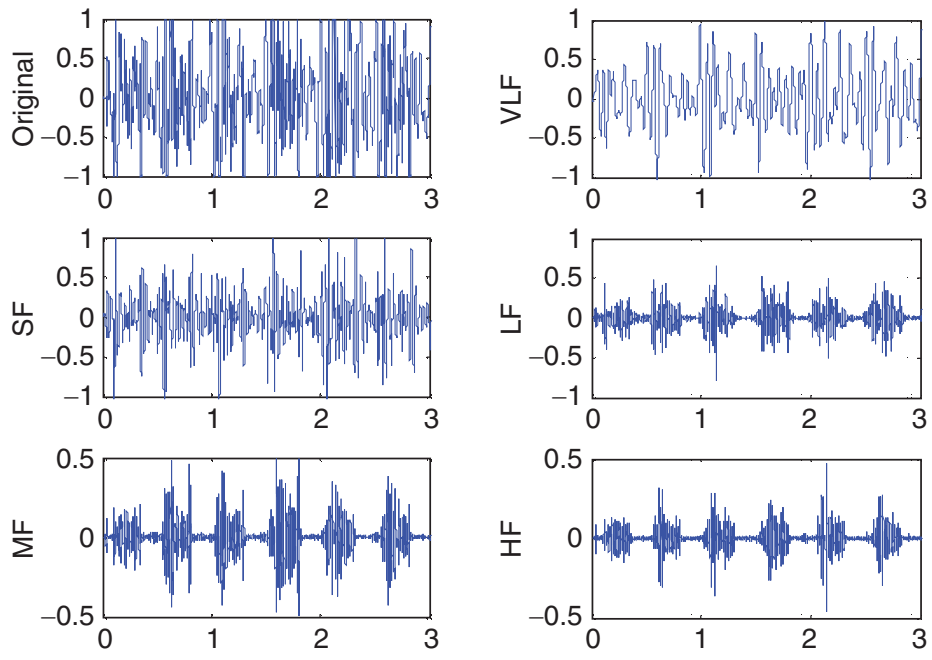

(d) TOF

Figure 2. HS signal examples of NHS, ASD, VSD, TOF and their reconstructed signals at different corresponding frequency band, Original, VLF, SF, LF, MF and HF. 

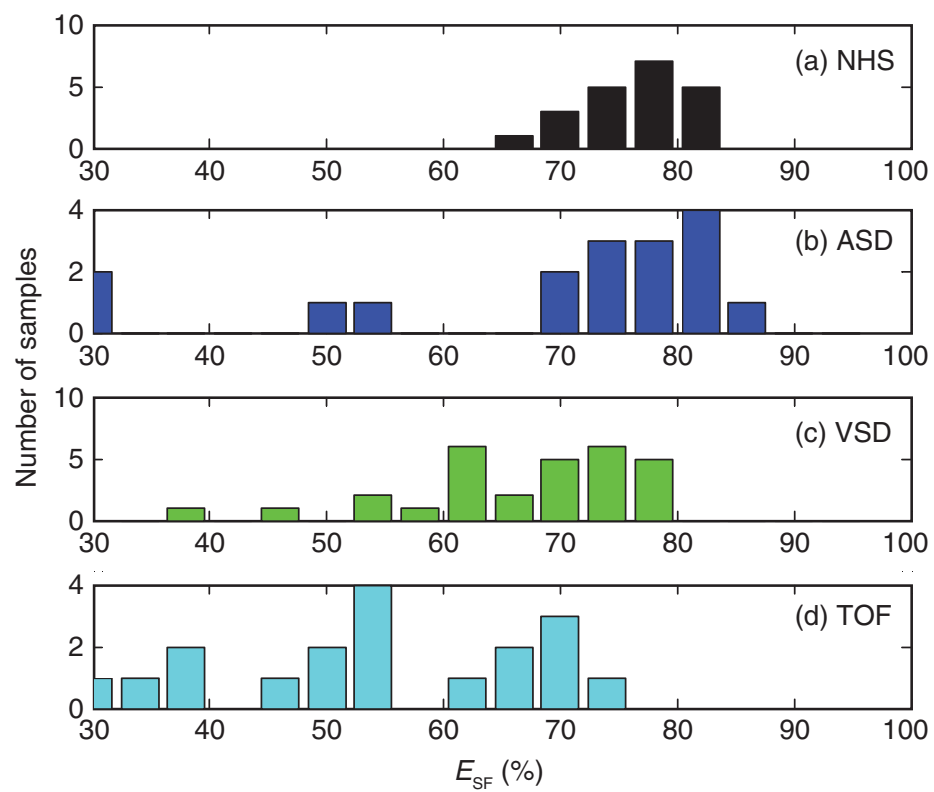

Figure 3. Energy distribution at SF frequency.
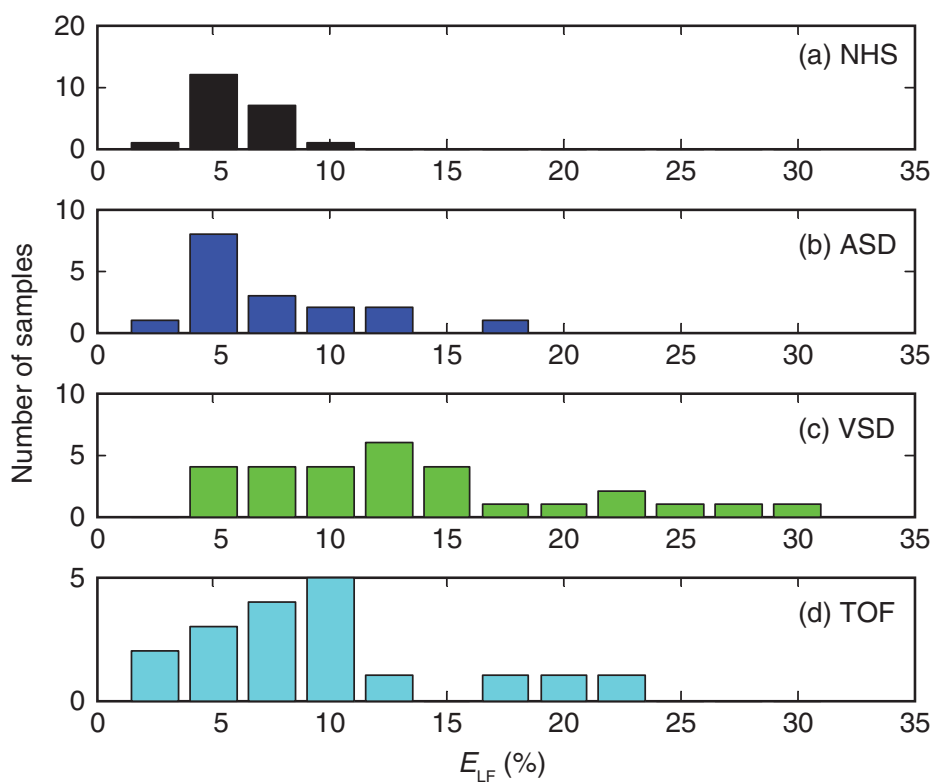

Figure 4. Energy distribution at LF frequency. 

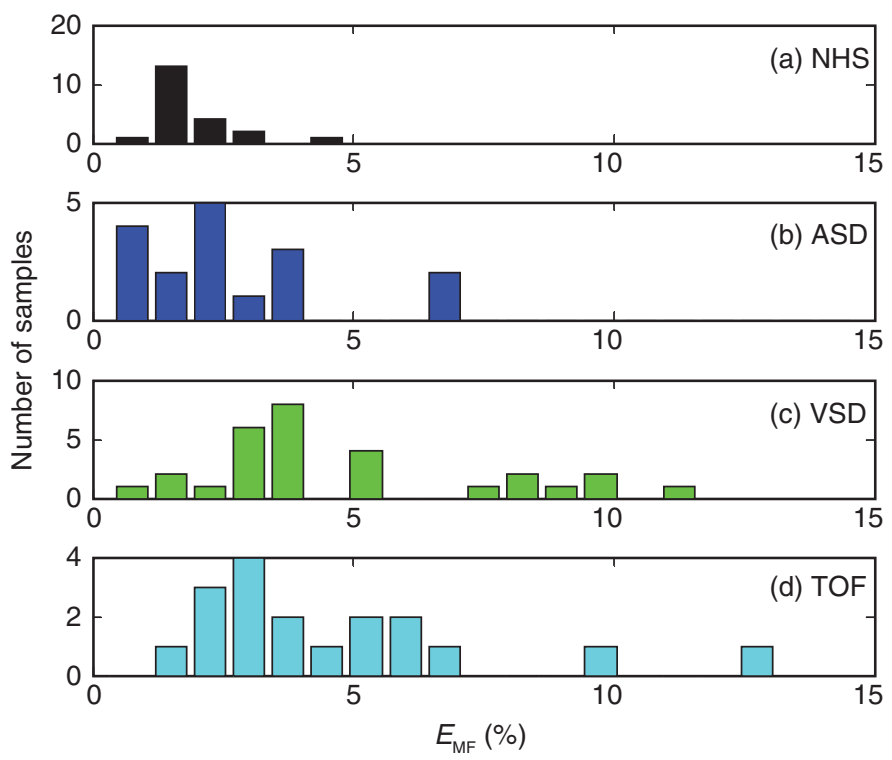

Figure 5. Energy distribution at MF frequency.
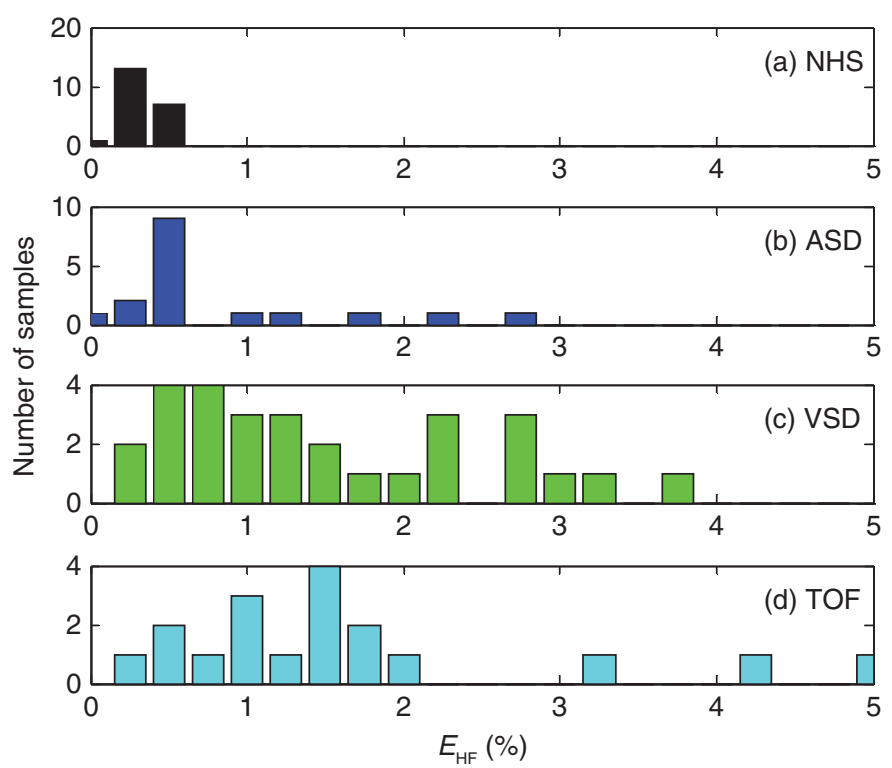

Figure 6. Energy distribution at HF frequency.

The averages and the standard deviations for these four conditions are summarized in Table 2. Statistical analysis was performed to show whether there is a significant difference in means among these four conditions (NHS, ASD, VSD and TOF) using the 
one-way ANOVA $F$-test. The very low $P$-values $(<0.0001)$ were taken as statistically significant. It is obvious that the distributions of NHS are following the normal probability distribution. The energy distributions of ASD are similar to NHS. Furthermore, the energy distributions of other CHDs are spread over a wide range and the energies maintain higher values at MF and HF bands compared to those in NHS.

Table 2 shows over $50 \%$ of energy in average concentrated at SF band for all conditions. This shows that the main component of HS is in the SF band. Further, Figure 2 and Table 2 indicate that the pathologic murmurs feature high energy distributions at LF, MF and HF.

On the other hand, the energy distribution at VLF band is considered to be a vibration or systaltic movement in the body due to the heartbeat. The energy distribution histograms at VLF band are shown in Figure 7. For NHS, the energy is

Table 2. Means and variances of the energy ratios at four frequency bands $(P<0.0001)$

\begin{tabular}{lccccc}
\hline $\begin{array}{l}\text { Group } \\
\left(\boldsymbol{E}_{\text {ave }} \pm \boldsymbol{E}_{\text {std }}\right)\end{array}$ & NHS & ASD & VSD & TOF & $P$-value \\
\hline$\left.E_{\mathrm{VLF}} \%\right)$ & $15.67 \pm 4.45$ & $20.30 \pm 19.18$ & $13.31 \pm 13.33$ & $30.69 \pm 19.84$ & $<0.0001$ \\
$E_{\mathrm{SF}}(\%)$ & $76.16 \pm 4.33$ & $68.67 \pm 18.95$ & $66.90 \pm 9.94$ & $53.45 \pm 13.63$ & $<0.0001$ \\
$E_{\mathrm{LF}}(\%)$ & $6.05 \pm 1.66$ & $7.51 \pm 3.89$ & $13.58 \pm 6.91$ & $9.60 \pm 5.41$ & $<0.0001$ \\
$E_{\mathrm{MF}}(\%)$ & $1.82 \pm 0.792$ & $2.65 \pm 1.76$ & $4.692 \pm 2.83$ & $4.58 \pm 2.86$ & $<0.0001$ \\
$E_{\mathrm{HF}}(\%)$ & $0.29 \pm 0.12$ & $0.84 \pm 0.74$ & $1.52 \pm 0.99$ & $1.68 \pm 1.26$ & $<0.0001$ \\
\hline
\end{tabular}
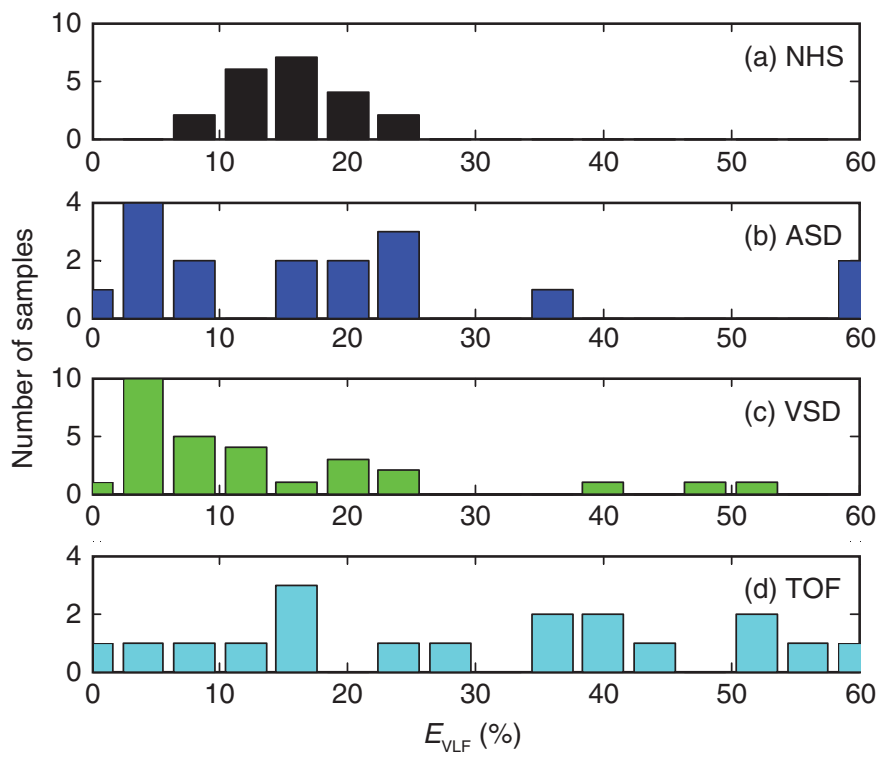

Figure 7. Energy distribution at VLF frequency. 
usually lower than $25 \%$ but for CHDs, the energies sometimes are higher than $30 \%$. It was found that the HSs recorded from the infant usually contain high energy components at VLF band and some of them account for more than $40 \%$ of the total energy. Because the infant has little fat, the pulsation of the heart easily leads to a movement of the whole chest region. This movement, especially due to the abnormal heart pulsation is captured by another stethoscope which shows the high energy component at VLF band. Since there are still many uncertainties for this VLF band, this study has mainly concentrated on the four bands, SF, LF, MF and HF.

\section{RESULTS AND DISCUSSIONS}

As discussed above, the main energy of HS is concentrated at SF band and the pathologic murmurs represent the high energy density at LF, MF and HF bands. Based on this fact and our investigation, the evaluation indexes of cardiac murmurs are proposed and defined as the following:

$$
I C M_{L}=\frac{E_{L}(\%)}{E_{S F}(\%)} \times 100 \%, \text { where } L=\{L F, M F, H F\}
$$

Figure 8 shows the plots of $I C M_{\mathrm{LF}}$ for NHS and CHDs. The average and standard deviation of $I C M_{\mathrm{LF}}$ for NHS are obtained as $8.01 \pm 2.44$. The threshold for cardiac murmurs $(\mathrm{TCM})$ at $\mathrm{LF}$ band is defined by $T C M_{\mathrm{LF}}=8.01+2.44=10.45$, depicted as a broken line in the figure. It means that it could be diagnosed to be the pathologic murmurs if the obtained energy level is higher than this threshold $T C M_{\mathrm{LF}}$. From Figure 8 , it is found that nine of the 17 ASD samples are below the threshold $T C M_{\mathrm{LF}}$, indicating nine subjects in false diagnosis. On the other hand, only four of the 29 VSD and three of the 18 TOF samples are below the threshold.

Point (A) in Figure 8 has the highest value of $I C M_{\mathrm{LF}}$ in NHS at LF band, which is beyond the threshold of $T C M_{\mathrm{LF}}$. This HS signal is further analysed and the results are plotted in Figure 9. It is confirmed that the strong energy component at LF band is the pulmonary sound due to a normal breath. The breath component can also be found at
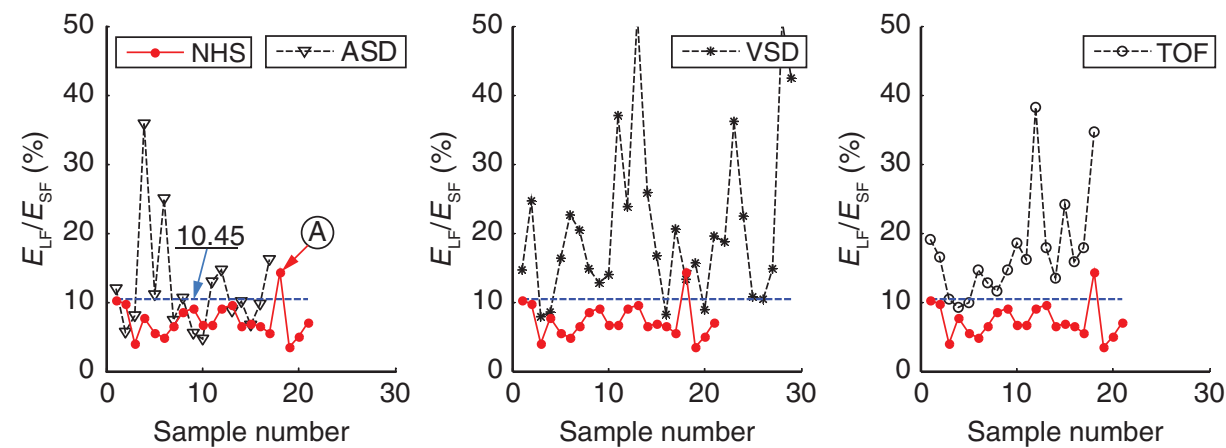

Figure 8. Distributions of the indexes of cardiac murmurs at LF band $\left(I C M_{\mathrm{LF}}\right)$. 
MF band but its intensity is much weaker than the one at LF band. General auscultation of HS, usually includes respiratory sound, and the pulmonary sound components are mainly distributed at LF and MF bands.

Regarding the MF band, Figure 10 shows the plots of $I C M_{\mathrm{MF}}$ for NHS and CHDs. The average and standard deviation of NHS's $I C M_{\mathrm{MF}}$ is $2.39 \pm 1.03$ and the threshold of the cardiac murmurs at MF band is set as $T C M_{\mathrm{MF}}=3.42$. It is found that there are 11 ASD, four VSD and no TOF samples below the level of $T C M_{\mathrm{MF}}$ indicating more than half ASD patients are misdiagnosed. However, the misdiagnosed number for TOF has been improved with the comparison of the results obtained at LF band.
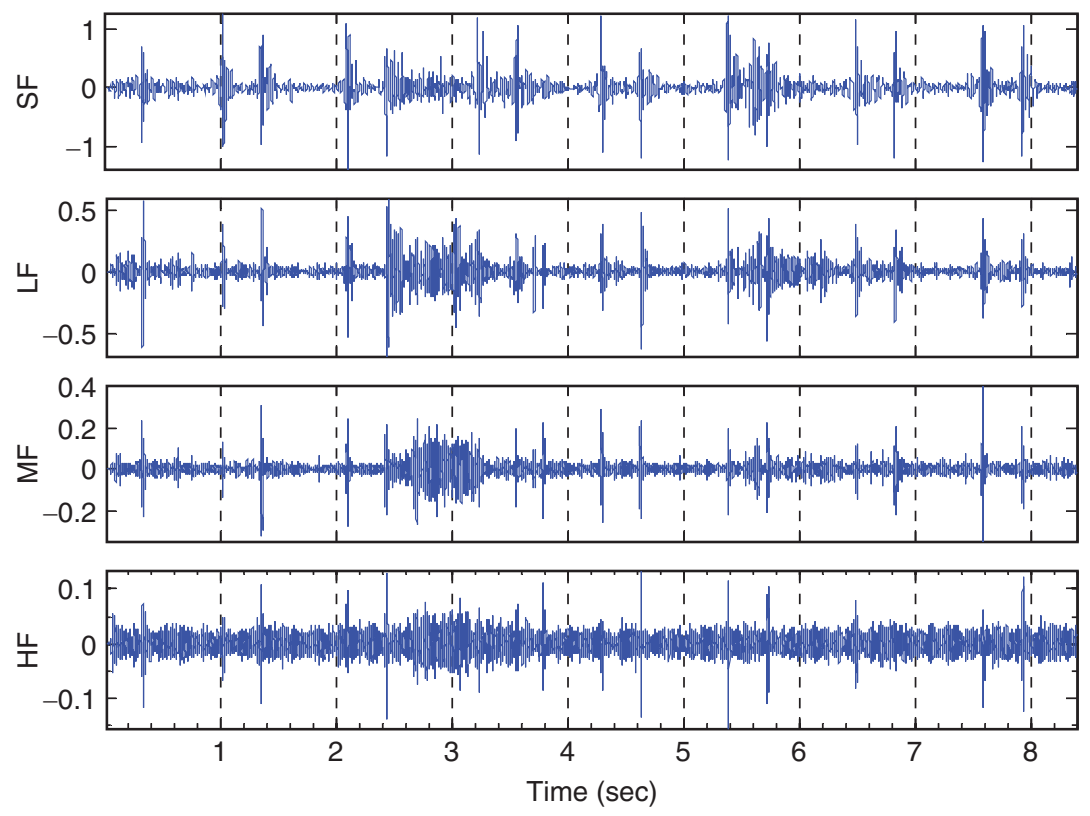

Figure 9. Example of pulmonary sound due to a normal breath, indicated by (A) in Figure 8 .
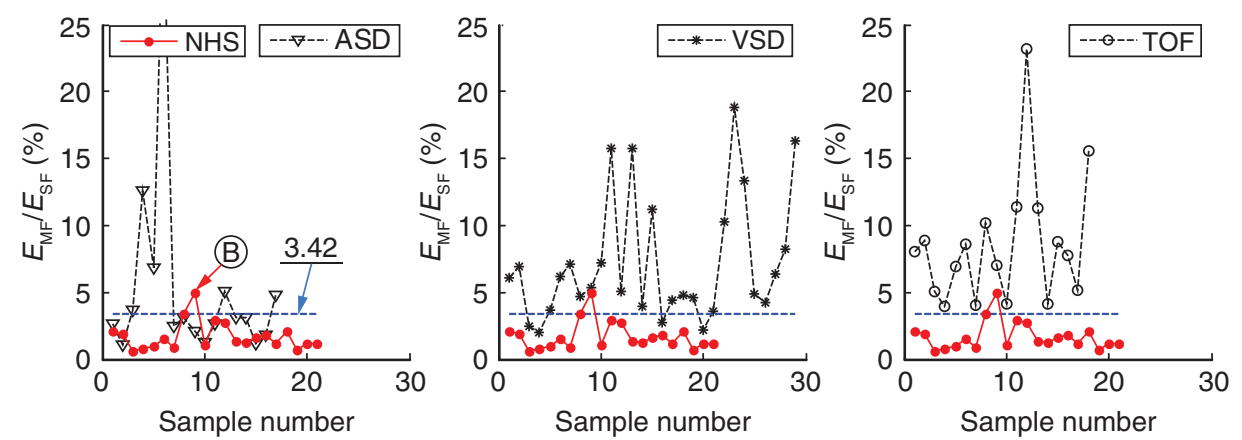

Figure 10. Distributions of the indexes of cardiac murmurs at MF band $\left(I C M_{\mathrm{MF}}\right)$. 
Point (B) in Figure 10 has the highest value of $I C M_{\mathrm{MF}}$ in NHS. Its time waveforms reconstructed at four expected frequency bands are plotted in Figure 11. It is clear that the breath sounds represent the strong components in LF, MF and HF bands. The energy distributions of the breath component have almost the same intensity at both LF and MF frequency bands.

The plots of $I C M_{\mathrm{HF}}$ are shown in Figure 12. The average and standard deviation of $I C M_{\mathrm{HF}}$ for NHS is $0.38 \pm 0.16$ and the corresponding threhold of the cardiac murmurs is given as $T C M_{\mathrm{HF}}=0.54$. The numbers of CHD samples below the threhold $T C M_{\mathrm{HF}}$ are two
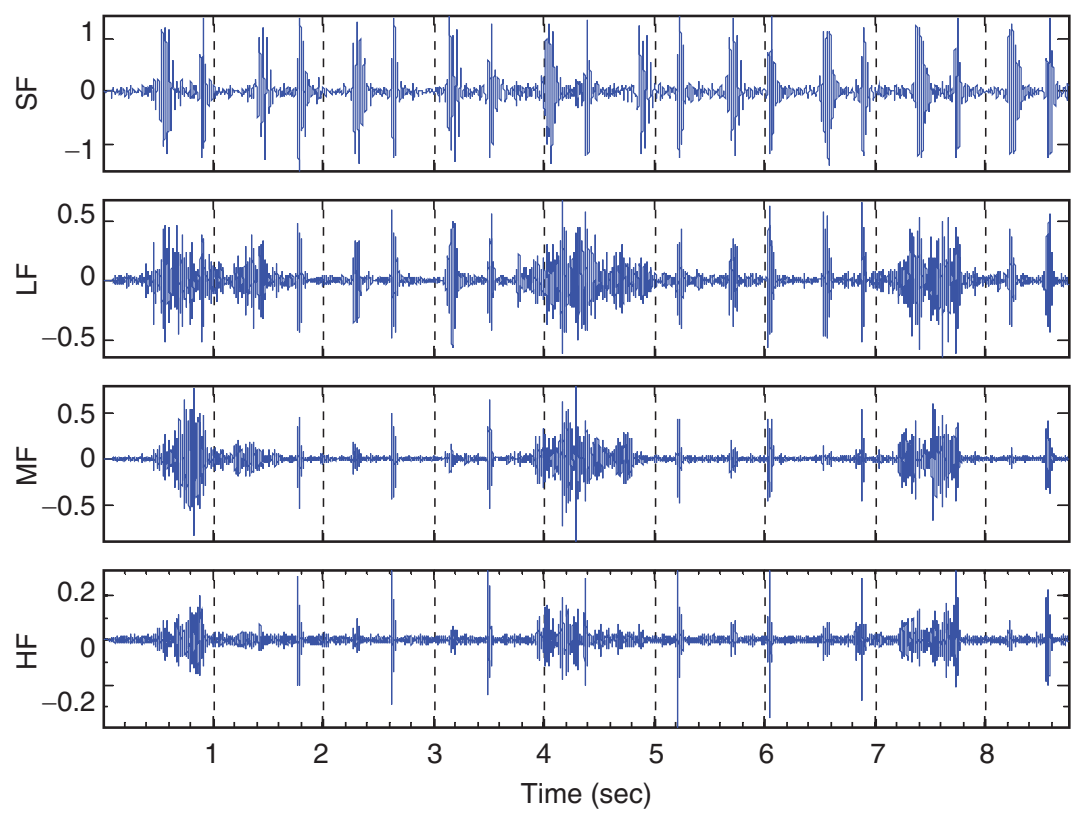

Figure 11. Example of pulmonary sound due to a normal breath, indicated by (B) in Figure 10.
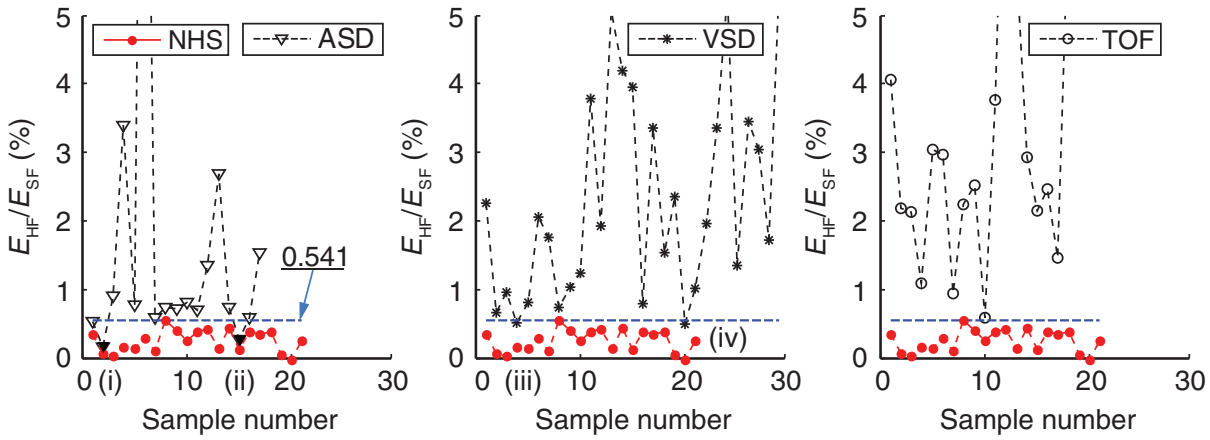

Figure 12. Distributions of the indexes of cardiac murmurs at $\mathrm{HF}$ band $\left(I C M_{\mathrm{HF}}\right)$. 
in $\operatorname{ASD}($ i,ii) $(\boldsymbol{\nabla})$, two in $\operatorname{VSD}($ iii,iv) (*) and none in TOF samples shown in Figure 12. The misdiagnosed cases are reduced significantly. This indicates that the cardiac murmurs evaluation index at $\mathrm{HF}$ band $I C M_{\mathrm{HF}}$ can be an evaluable index for quantitative diagnosis of HS murmurs. It is also evident that the diagnosis accuracy could be improved if the HS is recorded by a momentary stop of the breath, and in a quiet environment.

Based on the experimental investigation, the evaluation indexes of cardiac murmurs $\left(I C M_{\mathrm{LF}}, I C M_{\mathrm{MF}}\right.$ and $\left.I C M_{\mathrm{HF}}\right)$ are discussed. Performance measures such as True Positives (TP), False Negatives (FN), True Negatives (TN), and False Positives (FP), accuracy, sensitivity $(\mathrm{Sn})$ and specificity $(\mathrm{Sp})$ are evaluated. TN is the number of normal cases correctly identified as normal. FN is the number of CHD cases incorrectly identified as normal. TP is the number of CHD cases correctly identified as they are, and FP is the number of normal cases incorrectly identified as CHD. Sensitivity $(\mathrm{TP} /(\mathrm{TP}+\mathrm{FN}))$ is the ratio of $\mathrm{CHD}$ samples that are correctly identified to the total CHD. Specificity (TN/(TN+FP)) is the ratio of normal samples are correctly identified to total normal HS. Accuracy $(\mathrm{TP}+\mathrm{TN}) /(\mathrm{TP}+\mathrm{FN}+\mathrm{TN}+\mathrm{FP})$ which is the ratio of the number of correctly identified samples to total number of samples [25]. The performance measures for the evaluation indexes of cardiac murmurs are shown in Table 3. It can be seen that performance measures of $I C M_{\mathrm{HF}}$ yielded the highest sensitivity of $93.8 \%$ (the correct rate of CHD cases), specificity of $100 \%$ (the correct rate of normal cases) and accuracy of $95.3 \%$ (the correct rate of total cases), respectively, significantly better than those with $I C M_{\mathrm{MF}}$ and $I C M_{\mathrm{LF}}$.

As mentioned above, the energy distribution at VLF band is considered to be a vibration or systaltic movement in the body due to the heartbeat shown in Figure 7. In Figure 12, the four samples below the threshold of $T C M_{\mathrm{HF}}=0.54$ might be misdiagnosed as normal samples. Further analysis on these 4 CHD samples and comparing with NHS revealed that the energy ratio $E_{\mathrm{VLF}}$ of ASD(i), VSD(iii, iv) are over 20\%, which are higher than that of NHS (see Table 4). This means ASD(i) and VSD(iii,iv) can be distinguished from NHS by $E_{\mathrm{VLF}}(\%)$.

Therefore, only the energy distribution of ASD(ii) is close to NHS. However, based on the records of ASD(ii), the patient was diagnosed as a single ostium primum atrial septal defect without mitral regurgitation or left ventricle to right atrium shunting, while the other ASD cases in this study are ostium secundum atrial septal defects. Patients with smaller ostium primum atrial septal defects (little or no mitral regurgitation or left ventricle to right atrium shunting) are usually asymptomatic. Considering the analysis result of evaluation indexes $I C M_{\mathrm{HF}}$ and the four special cases of CHD (ASD(i,ii) and

Table 3. TN, FN, TP, FP, sensitivity (Sn), specificity (Sp) and accuracy (Acc) of evaluation indexes of cardiac murmurs $I C M_{\mathrm{L}}$

\begin{tabular}{lccccccc}
\hline $\boldsymbol{I C M _ { \mathbf { L } }}$ & TN & FN & TP & FP & Sn (\%) & Sp (\%) & Acc (\%) \\
\hline$I C M_{\mathrm{LF}}$ & 20 & 16 & 48 & 1 & 75 & 95.2 & 80 \\
$I C M_{\mathrm{MF}}$ & 20 & 15 & 49 & 1 & 76.6 & 95.2 & 81.2 \\
$I C M_{\mathrm{HF}}$ & 21 & 4 & 60 & 0 & $\mathbf{9 3 . 8}$ & $\mathbf{1 0 0}$ & $\mathbf{9 5 . 3}$ \\
\hline
\end{tabular}


Table 4. Energy ratios values at different frequency bands of CHD heart sound below the threhold $T C M_{\mathrm{HF}}$.

\begin{tabular}{lccccc}
\hline Sample No. & $\boldsymbol{E}_{\mathbf{V L F}}(\boldsymbol{\%})$ & $\boldsymbol{E}_{\mathbf{S F}}(\boldsymbol{\%})$ & $\boldsymbol{E}_{\mathbf{L F}}(\boldsymbol{\%})$ & $\boldsymbol{E}_{\mathbf{M F}}(\boldsymbol{\%})$ & $\boldsymbol{E}_{\mathbf{H F}}(\boldsymbol{\%})$ \\
\hline NHS $\left(E_{\text {ave }} \pm E_{\text {std }}\right)$ & $\mathbf{1 5 . 6 7 \pm 4 . 4 5}$ & $76.16 \pm 4.33$ & $6.05 \pm 1.66$ & $1.82 \pm 0.792$ & $0.29 \pm 0.12$ \\
ASD $(\mathrm{i})$ & $\mathbf{2 2 . 7 9}$ & 72.23 & 4.10 & 0.76 & 0.11 \\
ASD(ii) & $\mathbf{9 . 4 8}$ & 83.72 & 5.59 & 0.98 & 0.22 \\
VSD(iii) & $\mathbf{5 0 . 2 1}$ & 44.81 & 3.84 & 0.88 & 0.23 \\
VSD(iv) & $\mathbf{3 8 . 9 3}$ & 54.75 & 4.84 & 1.20 & 0.27 \\
\hline
\end{tabular}

VSD(iii, iv)) at VLF band, we can attain improved performance measures with sensitivity of $98.4 \%$, specificity of $100 \%$ and accuracy of $98.8 \%$, respectively.

\section{CONCLUSION}

CHDs are abnormalities in the heart's structure present at birth, which can range from mild to severe. Heart murmurs are pathological sounds produced by turbulent blood flow due to cardiac defects. This paper has described a new approach on analysis of the pathologic cardiac murmurs based on the WPD technique which analyses both low and high frequency sub-bands of HS signals, collected from clinical subjects in general environment. The HS signal was divided into five bands and the energy intensity at each frequency band was calculated and compared. Based on the analysis of clinic HS data, three evaluation indexes for cardiac murmurs were proposed for the analysis of the pathologic murmurs. Finally, the threshold values between the innocent and pathologic murmurs were determined based on the statistical results of the normal HSs. A case study on the NHS and CHD signals was performed to validate the usefulness and performance of the proposed method. The performance measures of $I C M_{\mathrm{HF}}$ yielded the highest sensitivity of $93.8 \%$, specificity of $100 \%$ and accuracy of $95.3 \%$, respectively. Furthermore, considering the analysis result of evaluation indexes $I C M_{\mathrm{HF}}$ at $\mathrm{HF}(344-1378 \mathrm{~Hz})$ and the four special cases at VLF band $(5-21 \mathrm{~Hz})$, we can obtain the improved performance measures with a sensitivity of $98.4 \%$, specificity of $100 \%$ and accuracy of $98.8 \%$, respectively.

\section{ACKNOWLEDGEMENTS}

This research was partially supported by the Japanese Ministry of Education, Culture, Sports, Science and Technology, Grant-in-Aid for scientific Research (C), 2009 2011, 21560243; 2012 2014, 24560261 and The Sichuan Provincial Key Technology R\&D Program(No. 2012GZ0019), China. We are grateful to Dr. Jinbao Zhang, Cardiothoracic Surgery, Chengdu Military General Hospital of People's Liberation Army, for his technical support and expert knowledge.

\section{CONFLICT OF INTEREST}

We confirm that there are no known conflicts of interest associated with this research. 


\section{REFERENCES}

[1] National Heart, Lung, and blood Institute, 2011. http://www.nhlbi.nih.gov/health/healthtopics/topics/chd/. Accessed June 14, 2014.

[2] Valafar H., Valafar F., Darvill A., Albersheim P., Kutlar A., Woods K.F., Hardin J., Predicting the effectiveness of hydroxyurea in individual sickle cell anemia patients, Artificial Intelligence in Medicine, 2000, 18(2): 133-148.

[3] CONGENITAL HEART DISEASE, 2011. http://www.pted.org/?id=list\#1. Accessed July 28, 2014.

[4] Amer Abdullah Lardhi, Prevalence and clinical significance of heart murmurs detected in routine neonatal examination, Journal of the Saudi Heart Association, 2010, 22(1): 25-27.

[5] Reichlin S., Dieterle T., Camli C., Leimenstoll B., Schoenenberger A. and Martina B., Initial clinical evaluation of cardiac systolic murmurs in the ED by noncardiologists, American Journal of Emergency Medicine, 2004, 71-75.

[6] Higuchi k., Sato K., Makuuch H., Furuse A., Takamoto S. and Takeda H., Automated diagnosis of heart disease in patients with heart murmurs: application of a neural network technique, International Journal of Medical Engineering and Technology, 2006, 30: 61-68.

[7] Sepideh Jabbari, Hassan Ghassemian, Modeling of heart systolic murmurs based on multivariate matching pursuit for diagnosis of valvular disorders, Computers in Biology and Medicine, 2011, 41(9): 802-811.

[8] Saeid Sanei, Mansoureh Ghodsi, Hossein Hassani, An adaptive singular spectrum analysis approach to murmur detection from heart sounds, Medical Engineering \& Physics, 2011, 33(3): 362-367.

[9] Samjin Choi, Youngkyun Shin, Hun-Kuk Park, Selection of wavelet packet measures for insufficiency murmur identification, Expert Systems with Applications, 2011, 38(4): 4264-4271.

[10] Yuerong Chen, Shengyong Wang, Chia-Hsuan Shen, Fred K. Choy, Matrix decomposition based feature extraction for murmur classification, Medical Engineering \& Physics, 2012, 34(6): 756-761.

[11] Zümray Dokur, Tamer Ölmez, Heart sound classification using wavelet transform and incremental self-organizing map, Digital Signal Processing, 2008, 18(6): 951-959.

[12] Fatemeh Safara, Shyamala Doraisamy, Azreen Azman, Azrul Jantan, Asri Ranga Abdullah Ramaiah, Multi-level basis selection of wavelet packet decomposition tree for heart sound classification, Computers in Biology and Medicine 2013, 43 (10): 1407-1414.

[13] M. Hariharan a,*, C.Y. Fook ${ }^{a}$, R. Sindhu ${ }^{\mathrm{b}}$, Abdul Hamid Adom ${ }^{\mathrm{a}}$, Sazali Yaacob ${ }^{\mathrm{a}}$, Objective evaluation of speech dysfluencies using wavelet packet transform with sample entropy, Digital Signal Processing 2013, 23 (3): 952-959.

[14] Shivnarayan Patidar, Ram Bilas Pachori, Segmentation of cardiac sound signals by removing murmurs usingconstrained tunable-Q wavelet transform, Biomedical Signal Processing and Control, 2013, 41(4): 559-567.

[15] Sanjay R. Bhatikar, Curt DeGroff, Roop L. Mahajan, A classifier based on the artificial neural network approach for cardiologic auscultation in pediatrics, Artificial Intelligent in Medicine, 2005, 33: 251-260.

[16] Abdulkadir Sengur, An expert system based on principal component analysis: artificial immune system and fuzzy k-NN for diagnosis of valvular heart diseases, Computers in Biology and Medicine, 2008, 329-338.

[17] Sepideh Babaei and Amir Geranmayeh, Heart sound reproduction based on neural network classification of cardiac vavle disorders using wavelet transforms of PCG signals, Computers in Biology and Medicine, 2009, 8-15.

[18] Amir A. Sepehri, Joel Hancq, Thierry Dutoit, Arash Gharehbaghi, Armen Kocharian, A. Kiani, Computerized screening of children congenital heart diseases, Computer Methods and Programs in Biomedicine, 2008, 92(2): 186-192.

[19] Samit Ari, Koushik Hembram, Goutam Saha, Detection of cardiac abnormality from PCG signal using LMS based least square SVM classifier, Expert Systems with Applications, 2010, 37(12): 8019-8026. 
[20] Samjin Choi, Zhongwei Jiang, Cardiac sound murmurs classification with autoregressive spectral analysis and multi-support vector machine technique, Computers in Biology and Medicine, 2010, 40 (1): 8-20.

[21] Shuping Sun, Haibin Wang, Zhongwei Jiang, Yu Fang, Ting Tao, Segmentation-based heart sound feature extraction combined with classifier models for a VSD diagnosis system, Expert Systems with Applications, 2014, 41(4): 1769-1780.

[22] Peter Libby MD, Robert O., Bonow MD, Douglas L., Mann MD FACC, Douglas P., Zipes MD, Braunwald's Heart Disease: A Textbook of Cardiovascular Medicine, 2007, p:1583.

[23] Samjin Choi, Detection of valvular heart disorders using wavelet packet decomposition and support vector machine, Expert System with Applications 2008, 35(4): 1679-1687.

[24] M. Nilsson, P. Funk, E.M. Olsson, B. vonSchele, N. Xiong, Clinical decision-support for diagnosing stress-related disorders by applying psychophysiological medical knowledge to an instance-based learning system, Artificial Intelligence in Medicine, 2006, 36: 159-176.

[25] Donna Giri ${ }^{\mathrm{a}}$, U. Rajendra Achary ${ }^{\mathrm{a}, \mathrm{c}, *}$, Roshan Joy Martis ${ }^{\mathrm{b}}$, S. Vinitha Sree ${ }^{\mathrm{d}}$, Teik-Cheng Lim ${ }^{\mathrm{a}}$, Thajudin Ahamed VI ${ }^{\mathrm{e}}$, Jasjit S. Suri ${ }^{\mathrm{f}, 1}$, Automated diagnosis of Coronary Artery Disease affected patients using LDA, PCA, ICA and Discrete Wavelet Transform, Knowledge-Based Systems, 2013,37: 274-282.

[26] T. Sawayama, Auscultation training by CD: Heart Sound, Publisher of Nankodo, 1994 (in Japan).

[27] K. Nakao, Online Bed Side Learning: Heart Sound Auscultation, available at, http://www.medic.mieu.ac.jp/student/sinnzou.html (in Japan). Accessed June 14, 2014. 



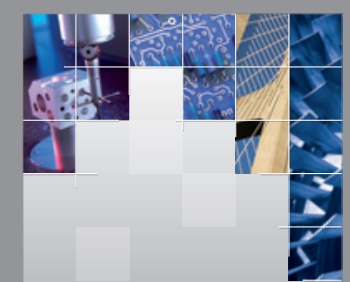

\section{Enfincering}
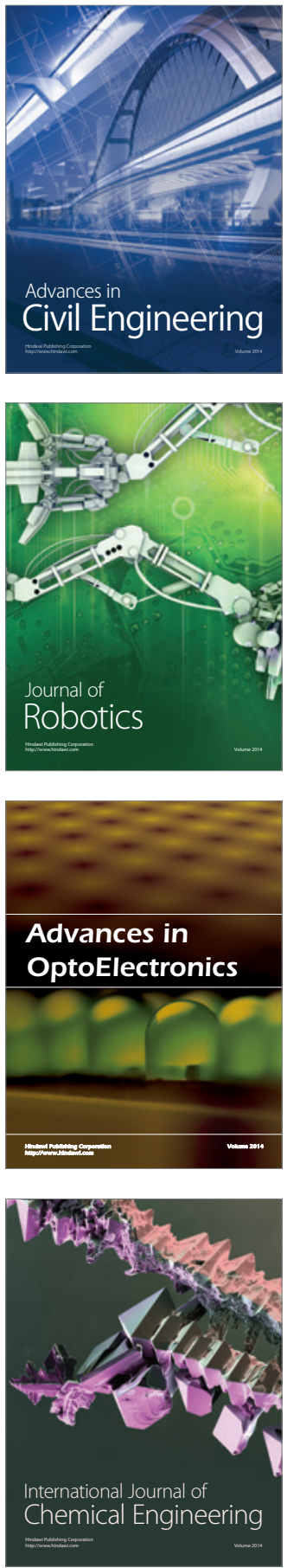

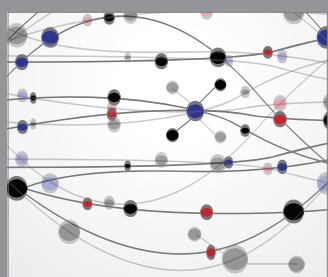

The Scientific World Journal

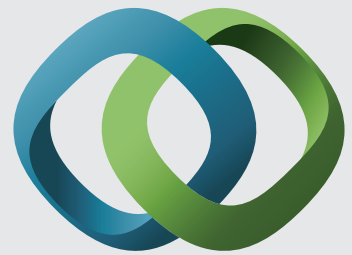

\section{Hindawi}

Submit your manuscripts at

http://www.hindawi.com
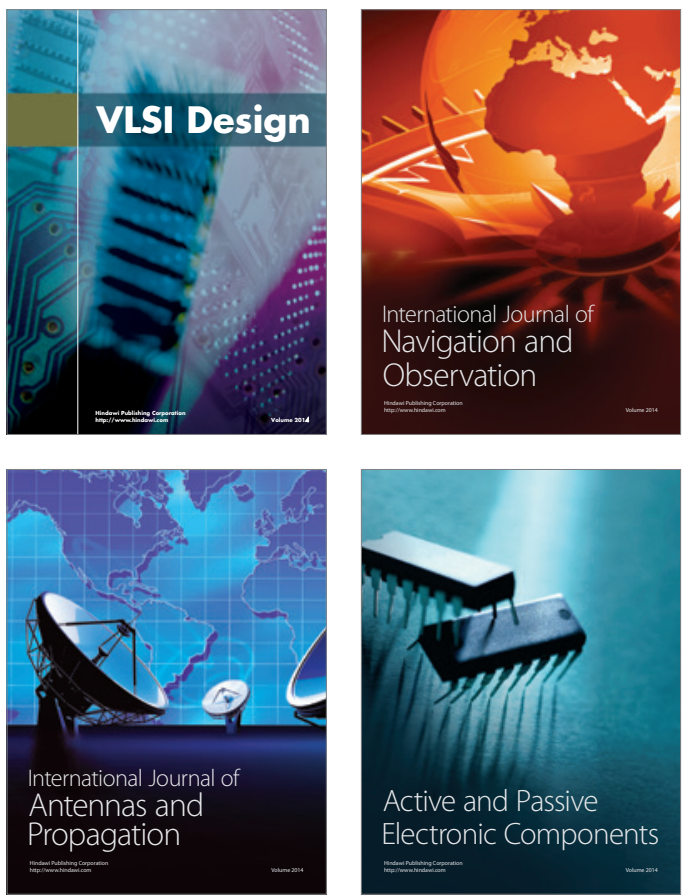
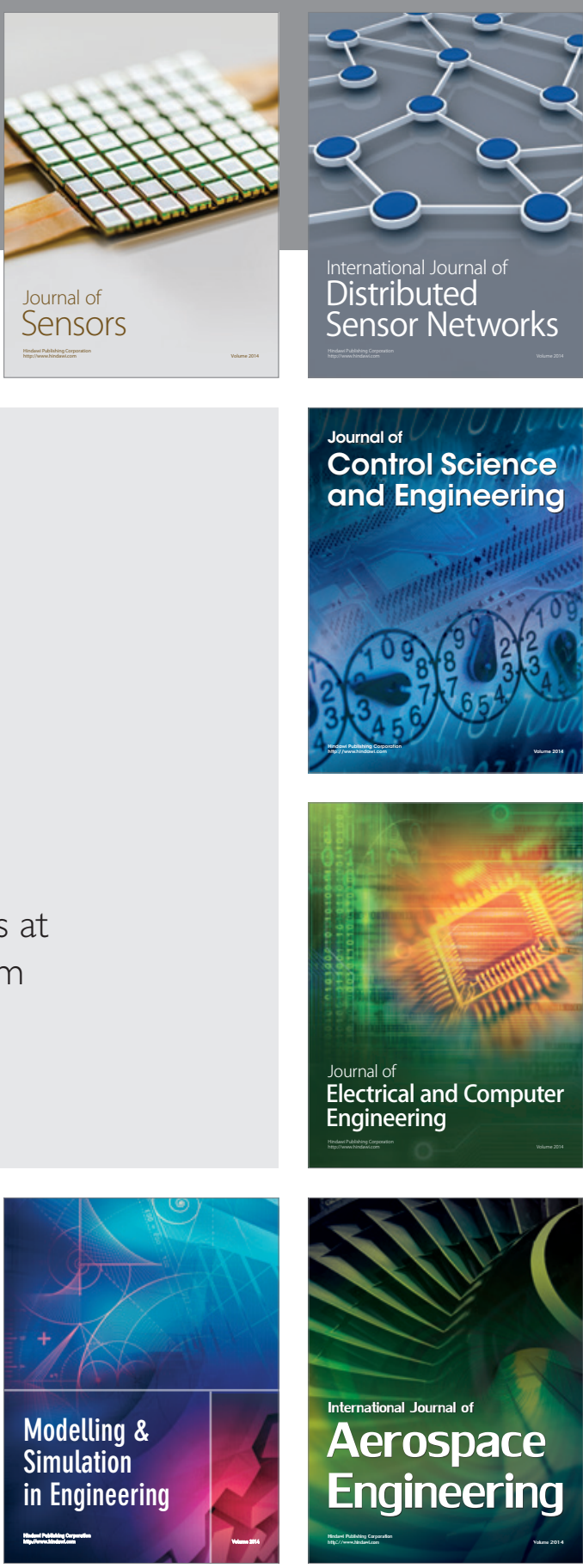

International Journal of

Distributed

Sensor Networks

Journal of

Control Science

and Engineering
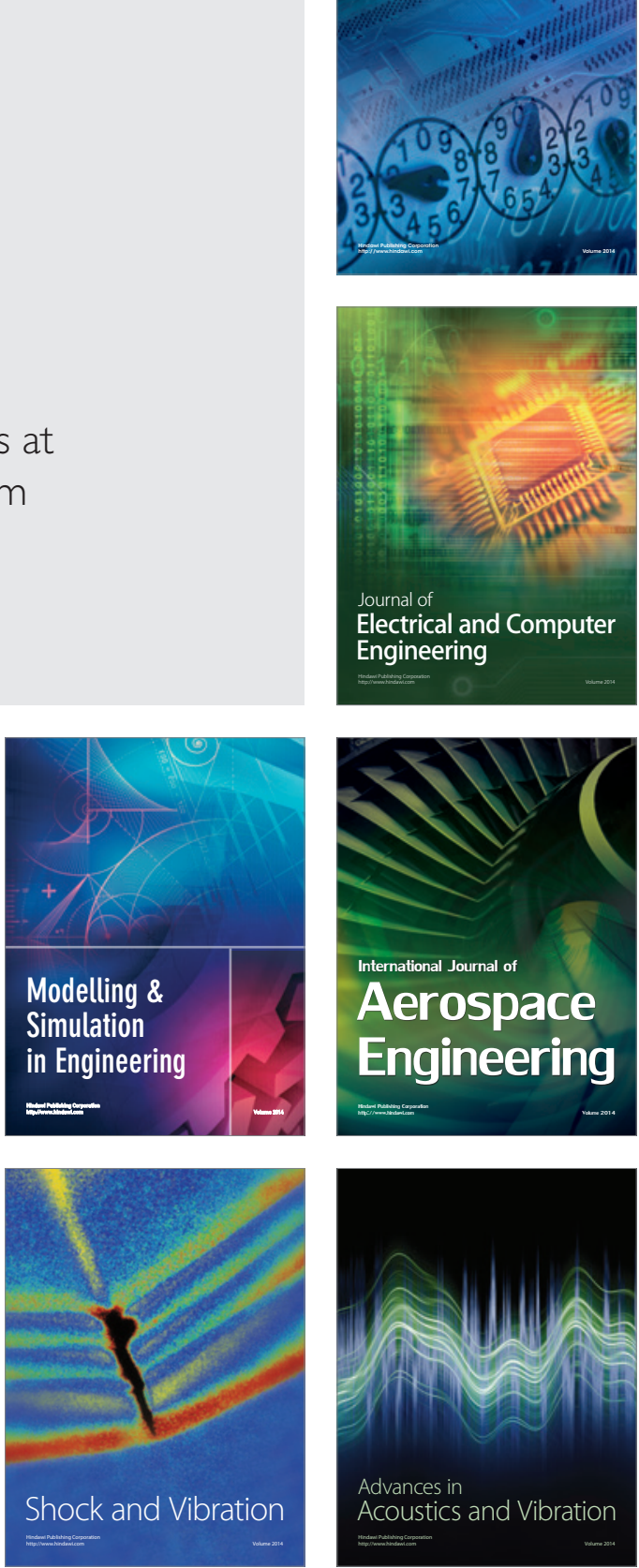